\title{
Deubiquitinase USP47-stabilized splicing factor IK regulates the splicing of ATM pre-mRNA
}

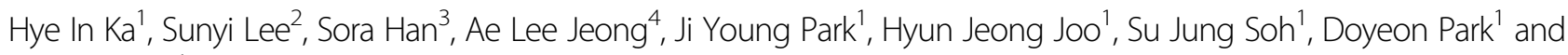
Young Yang ${ }^{1}$

\begin{abstract}
IK depletion leads to an aberrant mitotic entry because of chromosomal misalignment through the enhancement of Aurora B activity at the interphase. Here, we demonstrate that IK, a spliceosomal component, plays a crucial role in the proper splicing of the ATM pre-mRNA among other genes related with the DNA Damage Response (DDR). Intron 1 in the ATM pre-mRNA, having lengths $<200 \mathrm{bp}$, was not spliced in the IK-depleted cells and led to a deficiency of the ATM protein. Subsequently, the IK depletion-induced ATM protein deficiency impaired the ability to repair the damaged DNA. Because the absence of SMU1 results in IK degradation, the mechanism underlying IK degradation was exploited. IK was ubiquitinated in the absence of SMU1 and then subjected to proteolysis through the $26 \mathrm{~S}$ proteasome. To prevent the proteolytic degradation of IK, a deubiquitinating enzyme, USP47, directly interacted with IK and stabilized it through deubiquitination. Collectively, our results suggest that IK is required for proper splicing of the ATM pre-mRNA and USP47 contributes toward the stabilization of IK.
\end{abstract}

\section{Introduction}

Spliceosomes are large complex molecular machines that remove introns from transcribed pre-mRNAs. Spliceosomes, which are associated with more than 150 accessory proteins, are formed from five different small nuclear ribonucleoproteins (snRNP) ${ }^{1-5}$. In the first step of splicing, the U1 snRNP recognizes the $5^{\prime}$-splice site (SS) region and $\mathrm{U} 2 \mathrm{snRNP}$ is recruited to the branch site (BS) region by binding the $\mathrm{U} 2$ auxiliary factor (U2AF). The Tri-U4/U6.U5 snRNP is recruited to the U1 and U2 assembly and the helicase Prp28 helps the displacement of the U1 snRNP at the $5^{\prime}$-splice site into the U6 snRNA. Simultaneously, B-specific proteins, including IK and SMU1, are recruited to the B complex ${ }^{6,7}$. This B complex is transformed into an activated spliceosome $\left(\mathrm{B}^{\text {act }}\right)$ by unwinding of the U4/U6 RNA duplex triggered by the

\footnotetext{
Correspondence: Young Yang (yyang@sookmyung.ac.kr)

${ }^{1}$ Department of Biological Sciences, Sookmyung Women's University, Seoul 04310, Korea

${ }^{2}$ Drug Evaluation Group, R\&D Center CJ HealthCare, Icheon 04551, Korea

Full list of author information is available at the end of the article

These authors contributed equally: Hye In Ka, Sunyi Lee

Edited by: M. V. Niklison Chirou
}

helicase Brr2. The remaining U6 snRNP interacts with the U2 snRNA. During the course of B activation, B-specific proteins, including IK and SMU1, are dissociated. Because IK and SMU1 have no orthologs in yeast, these two proteins are considered to act as regulatory proteins ${ }^{8}$. In addition to this splicing function of IK and SMU1, IK plays a role in the cell cycle regulation, as reported previously ${ }^{9}$. IK localizes to the spindle pole and colocalizes with MAD1 at the spindle poles. The depletion of IK leads to a failure of the spindle pole localization of MAD $1^{10}$. The IK depletion is also able to disrupt the interaction between Aurora B kinase and protein phosphatase 2 $(\mathrm{PP} 2 \mathrm{~A})^{11}$, leading to aberrant mitotic entry with chromosomal misalignment and chromosomal instability ${ }^{12,13}$.

Posttranslational modification of spliceosomal proteins allows the exact splicing of pre-mRNAs through dynamic regulation of protein interactions ${ }^{14,15}$. Presently, it is known that the U4/U6.U5 tri-snRNP complex of spliceosome is subjected to ubiquitination for dynamic activation of the spliceosome. The splicing factor, Prp8, a component of the U5 snRNP, is ubiquitinated within triple snRNPs yielding the stabilization of the U4/U6-U5 
triple snRNP by repressing the U4/U6 unwinding ${ }^{16}$. PRP31, a component of the U4 snRNP, is modified with K63-linked ubiquitin chains by the PRP19 complex and is deubiquitinated by the ternary complex of USP15, SART3, and USP4. Thus, the ubiquitination and deubiquitination status of PRP31 regulates its interaction with the U5 snRNP component, PRP8, by stabilizing the U4/ U6.U5 tri-snRNP complex ${ }^{17}$. Ubiquitin has seven lysine residues. K11- or K48-linked ubiquitinated proteins are the targets of degradation by $26 \mathrm{~S}$ proteasome, whereas K63-linked ubiquitinated proteins mediate protein-protein interaction instead of proteolysis ${ }^{18-20}$. Until now, little is known about K11- or K48-linked ubiquitination of a spliceosomal component which might be relevant to regulation of splicing.

Activation of ataxia-telangiectasia mutated (ATM) kinase is an initial event in the DNA damage repair ${ }^{21-23}$. The activated ATM upon DNA damage leads to subsequent phosphorylation of downstream targets, including CHK1, CHK2, and $\mathrm{p} 53^{24-26}$. These phosphorylations promptly initiate the recruitment of damage repair factors at DNA lesions to repair DNA double-strand breaks (DSBs). Previous studies showed that ATM and spliceosome are reciprocally regulated ${ }^{27,28}$. When RNA polymerase II (RNAPII) encounters DNA lesions, snRNPs to form spliceosome are displaced and followed by facilitation of R-loop formation, which results in non-canonical activation of ATM to repair damage. On the other hand, the activation of ATM also was known to regulate alternative pre-mRNA splicing ${ }^{29}$. However, the splicing mechanism of ATM pre-mRNA by spliceosome is not yet clearly understood.

In the present study, we observed that the depletion of the splicing factor IK leads to intron 1 retention in ATM, but not in ATR, indicating that IK stabilization is very important for the proper splicing of ATM. In addition, we demonstrate that the stability of spliceosomal protein IK is regulated by ubiqutination-mediated proteolysis. USP47, which belongs to the ubiquitin-specific protease (USP) family of deubiquitinating enzymes (DUBs), prevents the proteolysis of IK through deubiquitination.

\section{Materials and methods \\ Cell culture}

HeLa and HEK 293T cells obtained from the ATCC were maintained in Dulbecco's modified Eagle's medium (DMEM, Hyclone) supplemented with $10 \%$ heatinactivated fetal bovine serum at $37^{\circ} \mathrm{C}$ in a humidified $5 \% \mathrm{CO}_{2}$ incubator, as described previously ${ }^{13}$. The cells were treated with the following DNA-damaging reagents: thymidine (Thy), mitomycin C (MMC), neocarzinostatin (NCS), camptothecin (CPT), etoposide (ETP), and hydroxyurea (HU). The cells were also treated with the protein synthesis inhibitor cycloheximide (CHX), the lysosomes inhibitor bafilomycin (Baf), the autophagy inhibitor wortmannin (Wor), and the proteasome inhibitor bortezomib (BTZ) or MG132.

\section{Plasmids}

Full-length human IK and USP47 cDNAs were purchased from OriGene (OriGene Technologies, Inc., Rockville, MD) and cloned into pcDNA 3.1, pcDNA 3.0, and pCMV-tag-2B vectors. In the restoration assay, IK cloned into $\mathrm{PCMV}$-tag$2 \mathrm{~B}$ vector was used. The full-length mouse IK $\mathrm{cDNA}$ was purchased from Origene and cloned into pcDNA 3.1 vector. Plasmid transfection was performed using a polyethylenimine (PEI) solution, X-tremeGENE HP DNA Transfection Reagent, and jetPRIME (Polyplus) reagent, according to the manufacturer's instructions.

\section{Antibodies}

Primary antibodies used for immunoblotting and immunofluorescence analyses were as follows: rabbit polyclonal anti-IK (Santa Cruz, sc-1335485), rabbit polyclonal anti-IK (Bethyl Laboratories, A301-708A), mouse monoclonal anti-USP47 (Santa Cruz, sc-100633), mouse monoclonal anti- $\beta$-actin (Santa Cruz, sc-47778), rabbit monoclonal anti-pATM S1981 (Cell Signaling, \#5883), rabbit monoclonal anti-ATM (Cell Signaling, \#2873), rabbit polyclonal anti-pATR S428 (Cell Signaling, \#2853), rabbit monoclonal ATR (Cell Signaling, \#13934), rabbit monoclonal anti-pCHK1 S345 (Cell Signaling, \#2348), rabbit polyclonal anti-pCHK1 S317 (Cell Signaling, \#2344), rabbit polyclonal anti-pCHK2 T68 (Cell Signaling, \#2661), mouse monoclonal anti-SMU1 (Santa Cruz, sc100896), mouse monoclonal anti-Ub (Santa Cruz, sc8017), mouse monoclonal anti-cleaved PARP (Cell Signaling, \#9546), rabbit polyclonal anti-Cleaved Caspase-3 (Asp175) (Cell Signaling, \#9661), rabbit monoclonal antiCleaved Caspase-9 (Asp315) (Cell Signaling, \#20750), rabbit monoclonal anti-Mre11(Cell Signaling, \#4847), rabbit polyclonal anti-pMre11(Ser676) (Cell Signaling, \#4859), rabbit polyclonal anti-Rad50 (Cell Signaling, \#3427), rabbit polyclonal anti-phospho p95 (Cell Signaling, \#3001), rabbit monoclonal anti-p95 (Cell Signaling, \#14956), rabbit monoclonal anti-phospho-Histone H2A.X (Ser139) (Cell Signaling, \#9718), mouse monoclonal antiHA (Santa Cruz, sc-7392), mouse monoclonal anti-FLAG (Sigma, F1804), mouse monoclonal anti-GFP (Santa Cruz, sc-9996), mouse monoclonal anti-SC-35 (Santa Cruz, sc53518), and mouse monoclonal anti-BrdU (Cell Signaling, \#5292) antibodies. The HRP-conjugated goat anti-mouse or anti-rabbit IgG (Fab) secondary antibodies were purchased from Enzo Life Sciences.

\section{RNAi}

For RNA interference assays, IK siRNA duplexes were designed to repress IK (\#1, 5'-CAAAGGUUGCAAGAU 
GUUU-3'; \#2, 5'-CUACCAAGGAGUUGAUCAA-3'; \#3, 5'-GCAUUCCAGUAUGGUAUCA-3'; \#4, 5' -AGACCAC ACUGACCACAAA-3'; \#5, 5'-AGCUGAGAUUGCCAG CAAA- $3^{\prime}$ ) and were used at a concentration of $20 \mathrm{nM}^{10}$. The SMU1 siRNA duplexes (5'-ACCACAGAAUGUUCA AAUA-3') and USP47 siRNA duplexes (\#1, 5'-GACUCU GAUAGUGUAGCAU-3'; \#2, 5'-GCUCAGAUCCCUUU GGCUATT-3'; \#3, 5'-GGCGUCAAGUCAACAUAUA TT $-3^{\prime}$ ) were also designed. The siRNAs were synthesized by Bioneer. For DUB siRNA screening, the Bioneer screening AccuTarget ${ }^{\mathrm{TM}}$ Human Ubiquitin siRNA set [SHS-0240] was used. The siRNAs were transfected into HeLa cells using Lipofectamine RNAiMax Transfection Reagent (Invitrogen) according to the manufacturer's transfection protocol. For IK restoration assays, the cells were transfected with a human or mouse IK-expressing plasmid, and then with the IK siRNA $18 \mathrm{~h}$ after transfection with the IK plasmid. The cell lysates were prepared $48 \mathrm{~h}$ after transfection with the IK siRNA.

\section{Immunofluorescence}

HeLa cells grown on coverslips were immediately permeabilized with $0.1 \%$ Triton X-100 in phosphate-buffered saline (PBS) for $3 \mathrm{~min}$ and were subsequently fixed with $4 \%$ paraformaldehyde in PBS for $10 \mathrm{~min}$. The cells were then washed twice with PBS, permeabilized with $0.5 \%$ NP40 in PBS for 5 min, and blocked with PBS-BT (3\% BSA and $0.1 \%$ Triton X-100 in PBS) for $30 \mathrm{~min}$ at room temperature. The cells on the coverslips were subsequently incubated with primary and secondary antibodies diluted in PBS-BT for $1 \mathrm{~h}$ at RT. The nuclei of the fixed cells were stained with Hoechst 33258 or DAPI mounting medium. Images were acquired on an LSM-700 Confocal Laser Scanning Microscope (Carl Zeiss) using a $\times 63$ oil immersion objective lens and ZEN software (Nikon).

\section{Immunoblot analysis}

For immunoblot analysis, cells were lysed in lysis buffer [50 mM Tris- $\mathrm{HCl}$ (pH 8.0), $150 \mathrm{mM} \mathrm{NaCl}, 1 \mathrm{mM}$ EDTA, and 1\% NP-40, supplemented with a protease and phosphatase inhibitor mixture (Roche)]. Cell lysates were obtained by centrifugation for $15 \mathrm{~min}$ at $4{ }^{\circ} \mathrm{C}$ at $20,000 \times g$, and concentrations of the supernatants were quantified using the Pierce BCA Protein Assay Kit (Thermo Scientific). Total protein lysates were prepared using $5 \times$ SDS sample buffer and heating at $99^{\circ} \mathrm{C}$ for $10 \mathrm{~min}$. Proteins were separated electrophoretically on a SDS-polyacrylamide gel and transferred onto a $0.45-\mu \mathrm{m}$ pore size nitrocellulose membrane. The membrane was incubated overnight with antibodies containing 3\% BSA in TBS-T ( $150 \mathrm{mM} \mathrm{NaCl}, 20 \mathrm{mM}$ Tris- $\mathrm{HCl}(\mathrm{pH} 8.0)$, and $0.05 \%$ Tween-20) at $4{ }^{\circ} \mathrm{C}$, followed by incubation with HRP-conjugated goat anti-mouse or anti-rabbit IgG (Fab) (Enzo Life Sciences) in 5\% skim milk in TBS-T at room temperature for $2 \mathrm{~h}$. Proteins were visualized with ECL western blotting reagent and analyzed on a Fusion Solo-S image analyzer (Vilber).

\section{Immunoprecipitation assay}

Cells were lysed with lysis buffer $[50 \mathrm{mM}$ Tris- $\mathrm{HCl}(\mathrm{pH}$ 8.0), $150 \mathrm{mM} \mathrm{NaCl}, 1 \mathrm{mM}$ EDTA, and $1 \% \mathrm{NP}-40$, supplemented with a protease and phosphatase inhibitor mixture (Roche)]. Cell lysates were obtained by centrifugation for $15 \mathrm{~min}$ at $4{ }^{\circ} \mathrm{C}$ at $20,000 \times g$. For endogenous protein immunoprecipitation, cell lysates were incubated with $3 \mu \mathrm{g}$ of antibody for overnight followed by incubation with protein $\mathrm{G}$ agarose beads (Amicogen, 2010005) for $2 \mathrm{~h}$ at $4{ }^{\circ} \mathrm{C}$. The immunocomplexes were then washed with lysis buffer for four times, and the immunocomplexes were separated by SDS-polyacrylamide gel and immunoblotting analysis was performed as described above.

\section{RNA immunoprecipitation}

For RNA immunoprecipitation, HeLa cells were resuspended in nuclear isolation buffer $(1.28 \mathrm{M}$ sucrose, $40 \mathrm{mM}$ Tris- $\mathrm{HCl} \mathrm{pH} 7.5,20 \mathrm{mM} \mathrm{MgCl}_{2}$, 4\% Triton X100), and nuclear protein was extracted using the RIP buffer. The anti-IK antibody was mixed with HeLa nuclear extract in the RIP buffer, and then incubated overnight at $4{ }^{\circ} \mathrm{C}$. Next, $30 \mu \mathrm{L}$ of protein G-agarose beads was added to each binding reaction, and the samples were further incubated at RT for $3 \mathrm{~h}$. The beads were subsequently washed five times with the RIP buffer, and RNA was extracted with TRIzol and reverse transcribed. The resulting CDNA was used as a template for PCR. The primers used for detecting ATM were designed to demonstrate that the detected signals were due to the RNA specifically binding to IK.

\section{Subcellular fractionation}

Cell fractionation was performed using the PARIS kit (Ambion), according to the manufacturer's instructions. The total isolated RNA was reverse transcribed using reverse transcriptase (Fermentas). The resulting cDNA was used as a template for PCR with the indicated primer sets. The values were normalized to those of GAPDH.

\section{ATM mini-gene splicing assay}

The 262-bp human ATM mini-gene construct was cloned into the pEGFP-N2 vector. The ATM fragment containing exon 1 with a 72-bp added start codon, 79-bp of intron 1 (containing the $5^{\prime}$-GU-A-AG-3', splicing recognition site), and 111-bp exon 2, flanked by the engineered EcoRI and BamHI restriction sites, was amplified from the cDNA extracted from the IK-depleted cells. The mini-gene construct $(0.5 \mu \mathrm{g})$ was transfected with $2 \mu \mathrm{L}$ of HP DNA transfection reagent (Roche), $24 \mathrm{~h}$ 
after siRNA transfection. After an additional $24 \mathrm{~h}$ incubation, protein was extracted using the lysis buffer and immunoblotted with an anti-GFP antibody, and the expression of green fluorescence protein (GFP) was observed by fluorescence microscopy.

\section{Metaphase spreads}

The cells were arrested by treating with colcemid (Sigma-Aldrich) at a concentration of $0.2 \mu \mathrm{g} / \mathrm{mL}$ for $90 \mathrm{~min}$, then harvested by incubating for $15 \mathrm{~min}$ at $37^{\circ} \mathrm{C}$ in $0.075 \mathrm{M} \mathrm{KCl}$, and subsequently fixed in a freshly prepared methanol:acetic acid $(3: 1 \mathrm{v} / \mathrm{v})$ solution. Metaphase spreads were prepared by dropping the cell suspension onto slides pre-wetted with $\mathrm{ddH}_{2} 0$. The slides were dried at $42^{\circ} \mathrm{C}$ for $60 \mathrm{~min}$ before staining with DAPI mounting medium (Sigma-Aldrich) in Gurr Buffer for $3 \mathrm{~min}$. After rinsing with fresh Gurr Buffer followed by rinsing with distilled water, the slides were fully dried and then monitored under a Zeiss microscope ${ }^{30}$.

\section{RT-PCR}

For RNA extraction, $500 \mu \mathrm{L}$ of the TRIzol reagent was directly added to cell culture plates, and the suspension was harvested and mixed with $100 \mu \mathrm{L}$ of chloroform. After centrifugation, $200 \mu \mathrm{L}$ of the clear supernatant was mixed with $200 \mu \mathrm{L}$ of $100 \%$ isopropanol and centrifuged at $12,225 \times g$ for $15 \mathrm{~min}$ at $4{ }^{\circ} \mathrm{C}$. The pellet was resuspended in $30 \mu \mathrm{L}$ of DNase/ RNase free DW (Invitrogen), and the concentration of total RNA was measured using an Epoch microplate. The RNA samples $(3 \mu \mathrm{g})$ were reverse transcribed using random hexamer primers. For quantification of band intensities, images from different sets of experiments were analyzed using the Image J software. The primer sequences used for RT-PCR analysis are available in Supplementary Table S1.

\section{Cell viability assay}

To assess the effects of DNA damage inducers on the viability of IK-depleted cells, HeLa cells were transfected with siIK \#1 for $24 \mathrm{~h}$, and the cells were plated in 48-well plates. CPT and ETP $(10 \mu \mathrm{M})$ were added to the cells $48 \mathrm{~h}$ after transfection, and the kinetics of the cells was monitored using an IncuCyte instrument (ESSEN BioSCIENCE).

\section{Annexin V staining}

To assess the cell death, the transfected HeLa cells were seeded onto 6-well plates, treated with trypsin-EDTA, centrifuged, and resuspended in PBS. Thereafter, the cell suspensions were incubated at room temperature with PI/ Annexin V for $15 \mathrm{~min}$ and analyzed using BD FACS Canto II. All the antibodies were purchased from eBioscience (eBioscience Inc. San Diego, CA).

\section{Statistical analysis}

Values are presented as the means \pm standard deviation (SD). Multiple comparisons within groups were performed by one-way analysis of variance (ANOVA), and differences between the means of individual groups were evaluated using Student's $t$-test. A value of $p<0.05$ was considered as the threshold for significant differences (" $p$ $<0.05,{ }^{* *} p<0.01,{ }^{* * * *} p<0.001$ ).

\section{Results \\ IK depletion results in abnormal fragmentation of chromosomes}

We have previously shown that IK depletion causes a marked increase in the proportion of mitotic cells showing misaligned chromosomes because of the increase in phosphorylation of Aurora A kinase, Aurora B kinase, and PLK $1^{11,13}$. In the present study, we classified the various aberrant chromosomal structures induced by the IK depletion. To this end, five different siRNAs targeting various regions of $\mathrm{IK}$, including the coding and $3^{\prime}$ untranslated regions (UTRs), were designed (Fig. 1a). All the five IK siRNAs successfully reduced the IK expression in HeLa cells and caused significant nuclear abnormalities (Fig. 1b). Because IK siRNA \#1 targeted the $3^{\prime}$-UTR, we used it for subsequent experiments including a rescue experiment. To classify the aberrant chromosomal structures, we examined the metaphase chromosome spreads after transfection with silK \#1 or silK \#3. The loss of IK resulted in various atypical chromosome shapes, including a parallel shape indicating cohesion defects and a fragmented shape indicating an impaired DNA repair system (Fig. 1c). Together, these results revealed that IK plays a crucial role in genome stability and stable cell viability in human cells.

\section{IK depletion inactivates the DNA damage-induced ATM signaling pathway via decrease in the ATM level}

Because IK depletion-induced chromosome fragmentation, we inferred that IK might be associated with the DNA damage repair system. To determine whether IK affects the DNA damage repair pathway under genotoxic stress, we treated the IK-depleted HeLa cells with neocarzinostatin (NCS), which causes DNA DSBs. In response to NCS treatment, IK-depleted cells showed a weak increase protein levels in ATM phosphorylation at S1981, CHK1 phosphorylation at S345, S317, and CHK2 phosphorylation at T68, but the levels of ATR protein and phosphorylation were not affected (Fig. 2a). To further confirm this result, the IK-depleted cells were also treated with other DNA damage inducers including thymidine (Thy), mitomycin C (MMC), camptothecin (CPT), etoposide (ETP), and hydroxyurea (HU), and showed a similar pattern with NCS treatment (Supplemental Fig. S1a, b). In addition, IK-depleted cells showed low percent 


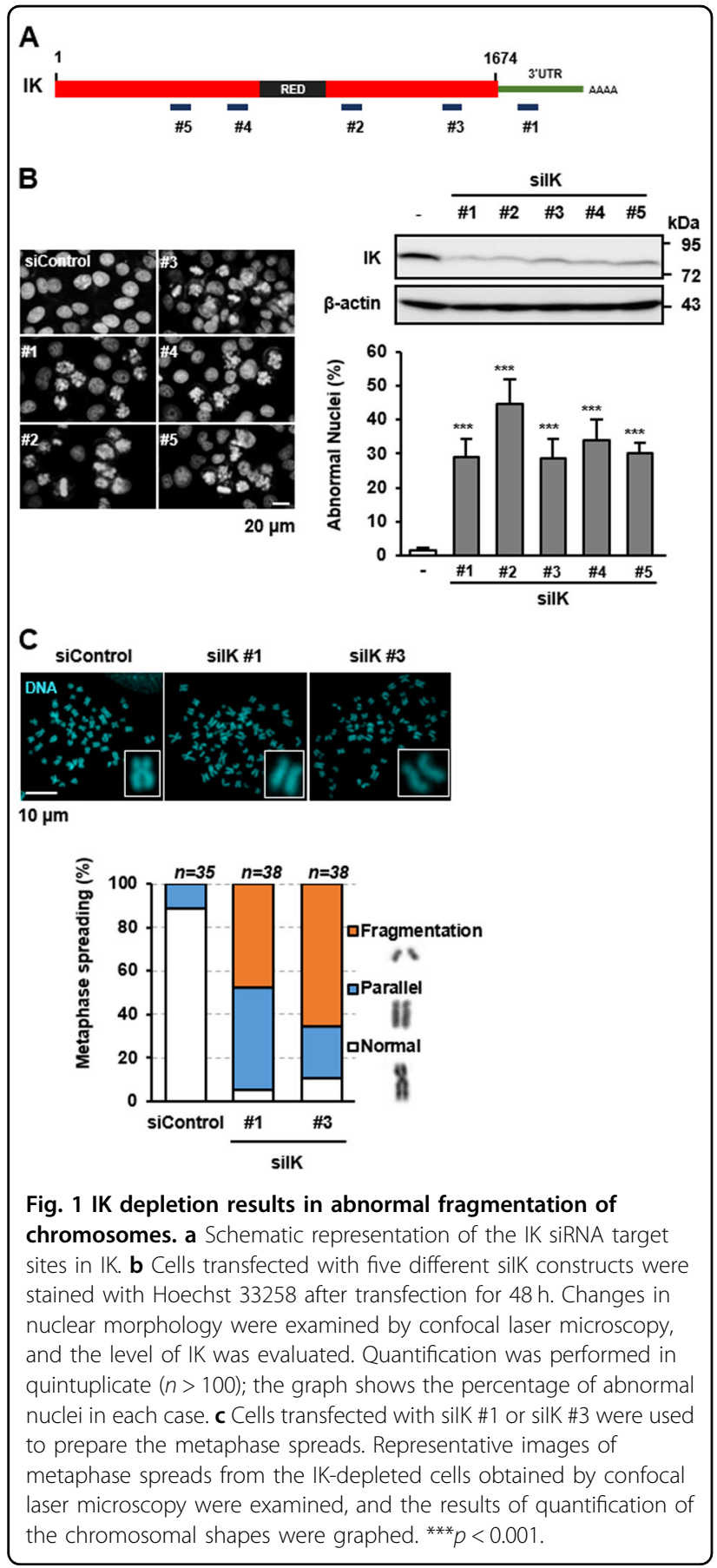

of co-localization between phosphorylated ATM and phosphorylation of the histone variant $\mathrm{H} 2 \mathrm{AX}(\gamma \mathrm{H} 2 \mathrm{AX})$ foci, which is formed when double-strand breaks appear upon HU treatment (Supplemental Fig. S1c, d), which is likely to due to lack of ATM amount. Next, to completely exclude the possibility that the decrease in ATM was due to an off-target effect of the IK siRNA, we transfected fulllength human or mouse IKs to IK-depleted cells, and the ATM protein level was almost restored in the IK- knockdown cells (Fig. 2b). Because IK depletion causes a decrease in the ATM protein levels, we hypothesized that the IK-depleted cells would exhibit an impairment of the ATM-mediated DNA damage repair. To examine this hypothesis, BrdU incorporation assay was performed to measure the frequency of ssDNA breaks in the IKdepleted cells. The IK-depleted cells showed a dramatic increase in the formation of BrdU foci (Fig. 2c). However, it is possible that the increase in BrdU incorporation would be due to DNA damage as well as checkpoint defect. To further prove this possibility, the IK-depleted cells were treated with CPT and ETP and showed significantly reduction in cell number (Fig. 2d).The DNA damage-inducing drugs treatment may affect the IKdepleted cells highly susceptible to decrease in cell proliferation. Collectively, these results indicate that IK is associated with the DNA damage repair system through the regulation of endogenous ATM protein levels.

\section{IK depletion decreases the spliceosomal excision of intron 1 in the ATM pre-mRNA}

Because IK functions as a splicing factor, it was determined whether the decrease of ATM protein levels in the IK-depleted cells was related to the aberrant function in spliceosomes. Thus, first, the levels of mRNA were examined using exon-exon specific primers designed based on different ATM exon regions and the level of $A T M$ mRNA was found to be decreased in the IK-depleted cells (Fig. 3a). Because previous report showed IK was related in splicing of short intron ${ }^{8}$, we also checked the intron sizes in ATM pre-mRNA using the human NCBI transcript reference sequences (Refseq: NM_000051) and displayed as a schematic view in ATM E1-E6 (Fig. 3b). We designed only primer targeting exon 1 and exon 2 region which is only short length in ATM pre-mRNA and performed RT-PCR. The intron 1 retention between exon 1 and exon 2 in the $A T M$ pre-mRNA was markedly increased in the IK-depleted cells (Fig. 3c), showing a ratio of $\sim 40 \%$ spliced intensity in ATM pre-mRNA (Fig. 3d). However, ATR splicing, which is responsible for the singlestrand DDR, was processed normally. In addition, $C D C 25 A, C D C 25 B$, and $C D C 25 C$, which are inhibited by the ATM-mediated pathway ${ }^{31}$, and the $E 2 F 1$ transcription factor, which increases the $A T M$ pre-mRNA levels ${ }^{32}$, were also normally processed without any intron retention. From these result, we confirmed that IK is a critical splicing factor involved in the processing of $A T M$ pre-mRNA. In addition, the intron retention of $A T M$ pre-mRNA splicing was slightly rescued when IK was re-expressed using a silK-resistant expression plasmid (Fig. 3e). There was no significant effect in $A T M$ splicing at exons 1-2 upon IK overexpression (Fig. 3f). Together, these findings suggest that IK depletion decreases the splicing of ATM pre-mRNA especially between exon 1 and 2 . 

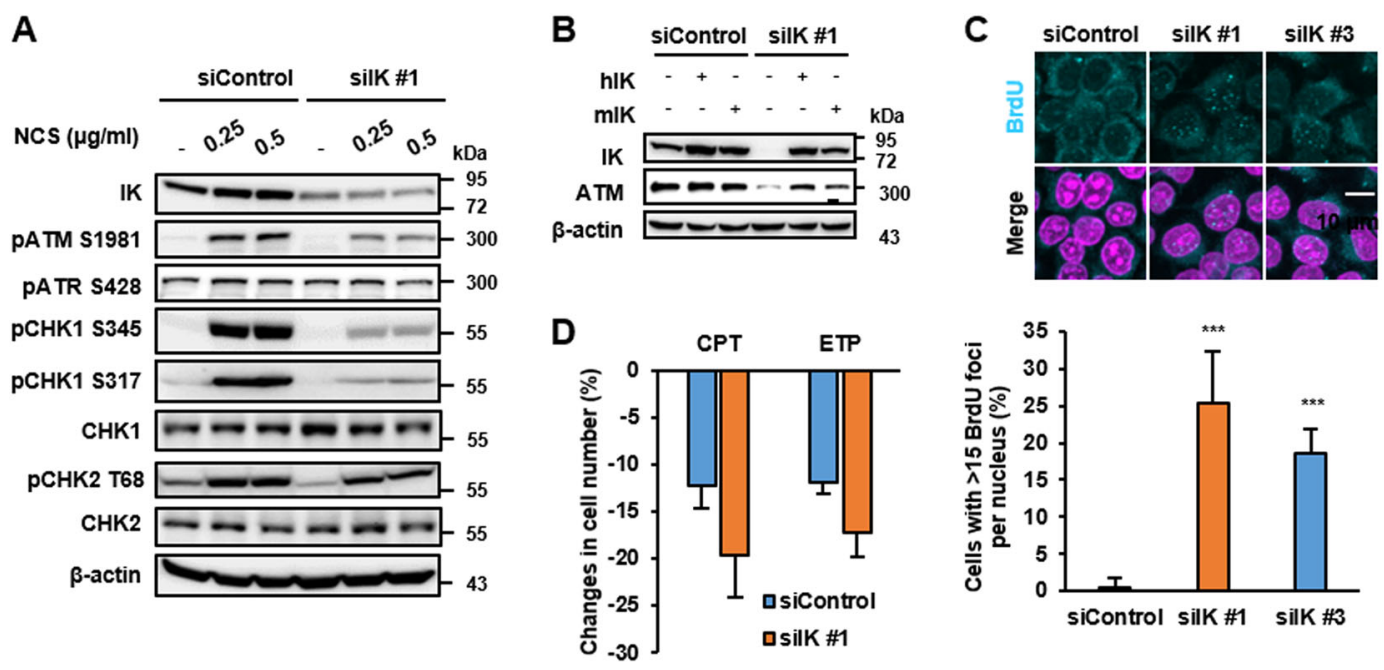

Fig. 2 IK depletion inactivates the DNA damage-induced ATM signaling pathway via decrease in the ATM level. a Cells transfected with silK $\# 1$ for $47 \mathrm{~h}$ were treated with neocarzinostatin (NCS) at the indicated concentrations for an additional $1 \mathrm{~h}$, and the levels of IK, pATM S1981, pATR S428, CHK1, CHK2, pCHK1 S345, pCHK1 S317, and pCHK2 T68 were examined. b Cells transfected with human IK or mouse IK for $18 \mathrm{~h}$ were transfected with silK \#1 for an additional $48 \mathrm{~h}$, and the levels of IK and ATM were examined. c Cells transfected with silK \#1 for $24 \mathrm{~h}$ were treated with BrdU at $100 \mathrm{\mu M}$ for an additional $24 \mathrm{~h}$ and stained with an anti-BrdU antibody. Representative images obtained by confocal laser microscopy were examined. The percentages of cells exhibiting more than $15 \mathrm{BrdU}$ foci per nucleus $(n>162)$ are graphed. ${ }^{* *} p<0.001$. d Cells transfected with silK \#1 for $48 \mathrm{~h}$ were treated with $10 \mu \mathrm{M}$ camptothecin (CPT) or etoposide (ETP) for an additional $24 \mathrm{~h}$. The cell growth was monitored for an additional $24 \mathrm{~h}$ using an IncuCyte live-cell imaging system. $n=3$.

IK depletion causes intron retention of ATM pre-mRNA and IK directly binds to the ATM pre-mRNA

Next, it was determined whether exon skipping is a possible event in the IK-depleted cells and the region between exons 1 and 2 was examined. In IK-depleted cells, intron 1 retention was observed without exon skipping (Fig. 4a). To further validate the intron 1 retention, we constructed an ATM mini-gene containing exon 1-intron 1-exon 2 positioned upstream of the GFPcoding DNA (Fig. 4b). The IK-depleted cells failed to remove the intron 1 . Thus, the level of GFP expression was markedly decreased, owing to a premature stop codon arising from a reading frame shift resulting from intron 1 retention in the absence of IK (Fig. 4b). Furthermore, we examined the RNA levels of ATM in the cytoplasmic and nuclear fractions of IK-depleted cells to determine whether intron retention- $A T M$ transcripts was exported to the cytoplasm. Most of the intron 1-retaining forms of $A T M$ transcript accumulated in the nuclear fraction, without being exported to the cytoplasm (Fig. 4c). Next, to confirm whether the ATM transcript localizes at the spliceosome containing $\mathrm{IK}$, the co-presence of ATM transcript and IK protein in the nuclear extracts was examined. For confirmation of their co-presence, we precipitated nuclear extracts of HeLa cells with an anti-IK antibody and used the precipitates to amplify the ATM E1-E2 pre-mRNA primer. The amplified $A T M$ bands were observed in the anti-IK precipitates but not in the control IgG precipitates (Fig. 4d).
IK is degraded by the proteasome complex in the absence of SMU1

The interaction between SMU1 and IK is essential for their spliceosomal functions and depletion of SMU1 or IK causes the changes in the expression level and alternative splicing profiles of several genes with intron specific effect $^{33}$. We confirmed an endogenous interaction of IK with SMU1 using a pull-down assay (Fig. 5a). Next, we have examined the localization of IK within nuclear speckles where splicing factors are stored when not used in splicing by co-staining with SC35. The IK was costained with SC35 (Supplementary Fig. S2) and disappeared during mitosis (Fig. 5b). When SMU1 was depleted, the level of the IK was reduced (Fig. 5c). Thus, SMU1 depletion resulted in a decrease in the ATM total and phosphorylation protein level as well as in the IK expression level (Fig. 5d). To investigate whether SMU1 depletion decreases the efficiency of $A T M$ pre-mRNA splicing as observed in the case of IK depletion, the splicing of the ATM pre-mRNA was examined in the SMU1depleted cells. The loss of SMU1 increased intron retention between exon 1 and exon 2 similar to that observed with IK depletion (Fig. 5e), suggesting that ATM premRNA splicing is regulated by IK which is stabilized by SMU1 binding. Next, to determine how SMU1 affects the stability of the IK protein, the SMU1-depleted cells were treated with bafilomycin (Baf) to inhibit the lysosomes (Fig. 5f), wortmannin (Wor) to inhibit the autophagy (Fig. $5 \mathrm{~g}$ ), and bortezomib (BTZ) to inhibit the proteasome (Fig. 


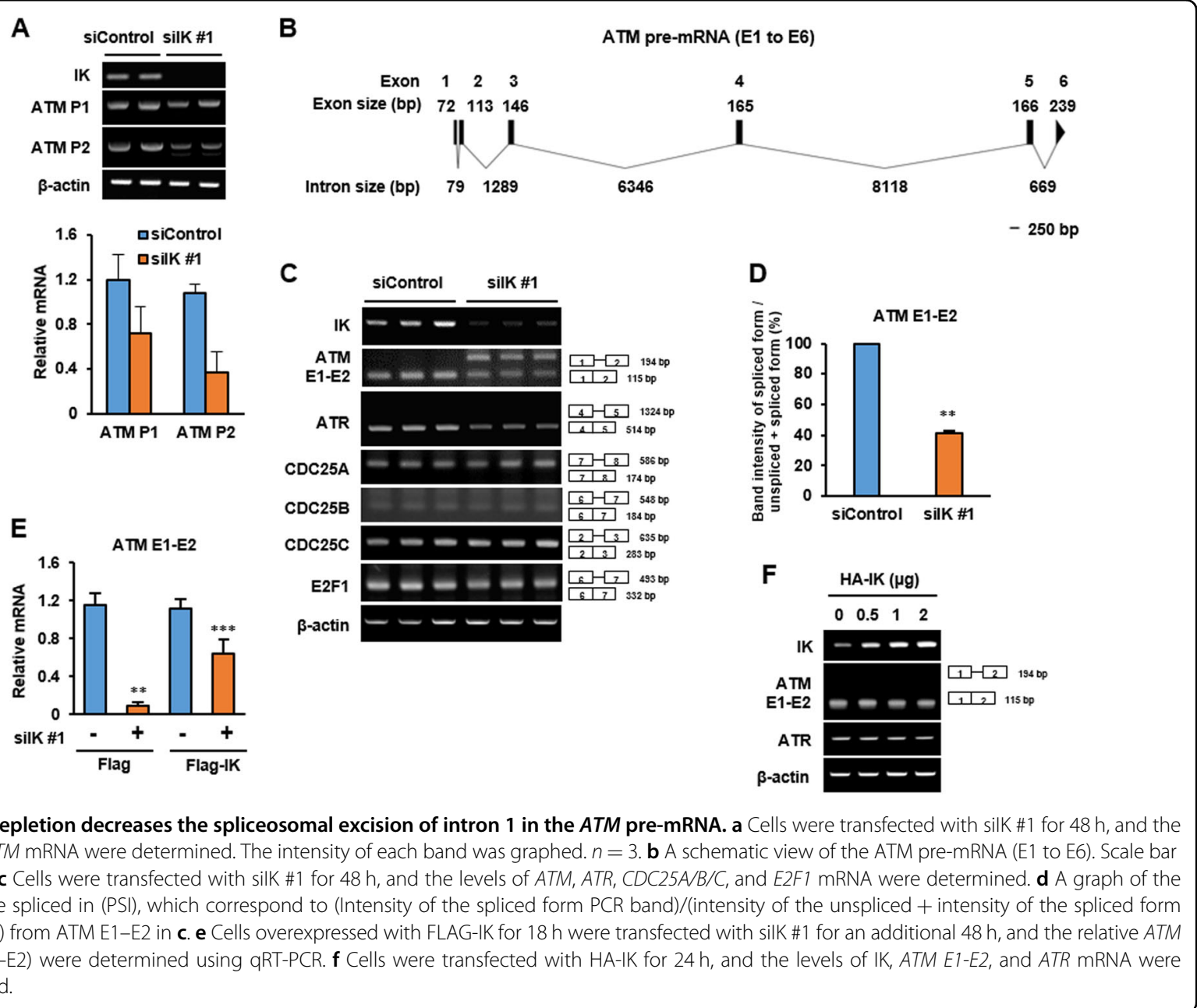

5h). When treated with BTZ, degradation of IK was only inhibited in SMU1-depleted cells. Moreover, we observed that another representative proteasome inhibitor, MG132 treatment also inhibited the IK degradation induced by SMU1 depletion (Fig. 5i). To further confirm this observation, we examined the ubiquitination of IK in cells overexpressing SMU1. The overexpression of SMU1 inhibited the ubiquitination of IK (Fig. 5j), indicating that SMU1 is required for the stabilization of IK. Taken together, these data shows IK is degraded by the proteasome complex in the absence of SMU1.

\section{USP47 is a candidate deubiquitinase for IK}

Because IK is regulated by a ubiquitin-dependent mechanism, we further investigated as to which deubiquitinating enzymes (DUBs) are able to stabilize IK. To identify the DUBs that could regulate the IK stability, the IK protein levels were determined after the treatment of siRNAs targeting each of the 76 DUBs (Fig. 6a; Supplementary Fig. S3). Among the 76 DUBs, 11 that are known to associate with DDR were separately examined because IK may play a role in the DNA damage repair (Fig. 6b). The USP47 depletion consistently decreased the endogenous levels of the IK protein. Next, USP47 was depleted with three different siRNAs targeting different regions of the USP47 (Fig. 6c). Among three siRNAs, siRNA\#1 targeting the $3^{\prime}$-UTR was most effective and was, therefore, used in subsequent experiments. The USP47-depleted cells showed a decrease in the levels of both IK and SMU1 (Fig. 6d) without any decrease in the IK mRNA levels (Fig. 6e). Moreover, the USP47 overexpression rescued the levels of IK and SMU1 in the USP47-depleted cells (Fig. 6f). Next, to examine whether USP47 directly binds to IK, cell lysates expressing HA-IK and Flag-USP47 were used in a pull-down assay with anti-HA or -Flag antibodies. USP47 and IK were observed in both the immunoprecipitates (Fig. 6g, h). In addition, cell lysates obtained without the overexpression of HA-IK and Flag-USP47 were immunoprecipitated with the anti-USP47 monoclonal antibody. The endogenous IK was also included in the immunoprecipitates (Fig. 6i). Taken together, these results imply that USP47 should be a deubiquitinase responsible for the regulation of the endogenous IK. 

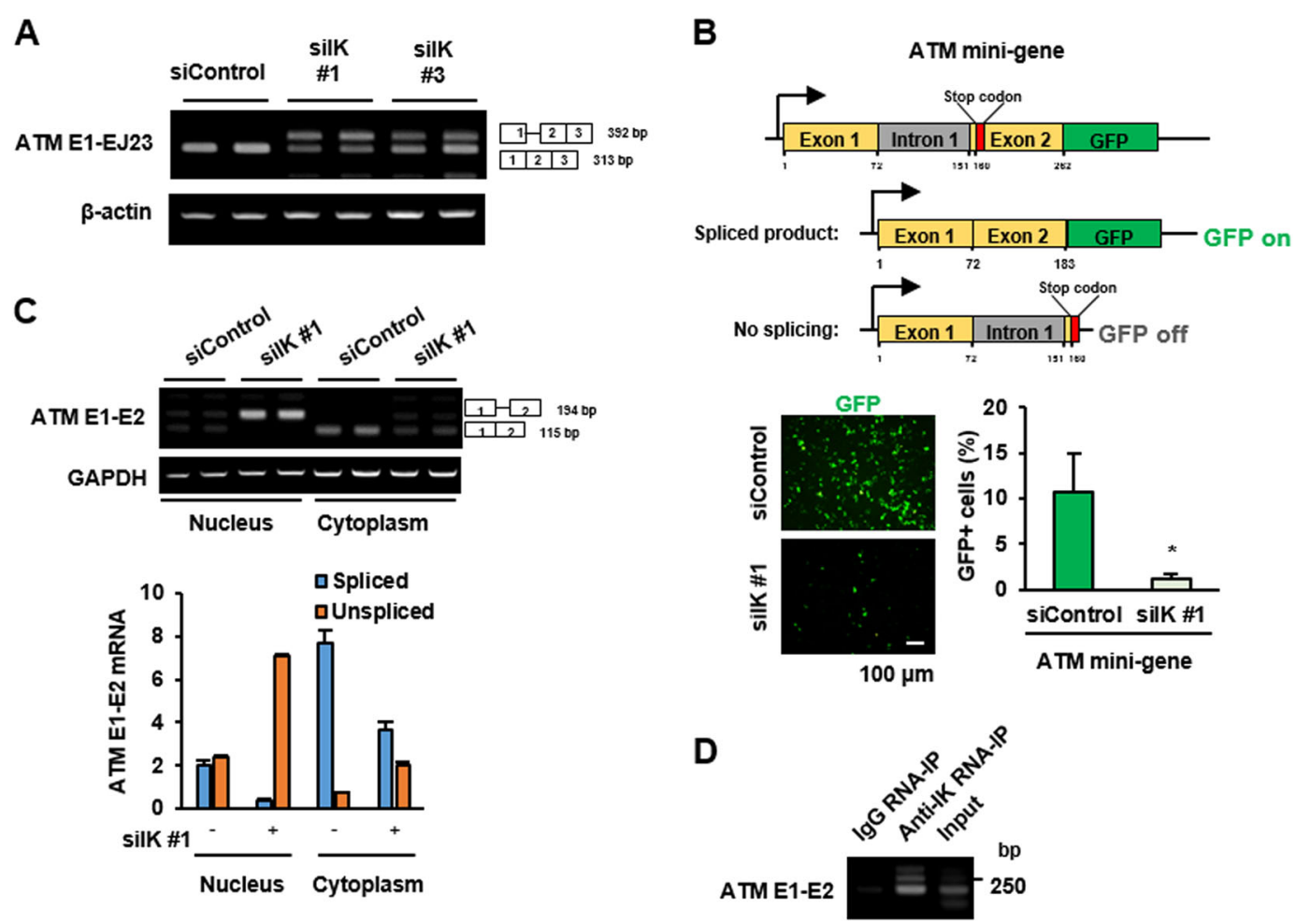

Fig. 4 IK depletion causes intron retention of ATM pre-mRNA and IK directly binds to the ATM pre-mRNA. a Cells were transfected with silK $\# 1$ or silK \#3 for $48 \mathrm{~h}$, and the levels of ATM E1-EJ23 mRNA were determined. b Cells transfected with silK \#1 for $24 \mathrm{~h}$ were transfected using the GFPATM-mini-gene construct for an additional $24 \mathrm{~h}$, and the levels of IK and GFP were examined. Representative images of green fluorescent proteinexpressing cells obtained using fluorescence microscopy were examined, and the intensity of green fluorescence was quantified. $n=3 .{ }^{*} p<0.05$. $\mathbf{c}$ Cells were transfected with silK \#1 for $48 \mathrm{~h}$, and cytoplasmic RNA and nuclear RNA were extracted separately. The levels of ATM mRNA were examined, and the intensity of each band was graphed. ${ }^{*} p<0.05$. $\mathbf{d}$ HEK-293T cells were immunoprecipitated with anti-IK antibodies, and the levels of ATM mRNA were determined.

\section{USP47 stabilizes IK through deubiquitination}

To further confirm the role of USP47 as a deubiquitinase of IK, the USP47-depleted cells were treated with an inhibitor of protein translation cycloheximide (CHX), to examine the IK stability. The USP47-depleted cells showed markedly reduced half-life of the endogenous IK compared to that in the non-depleted cells (Fig. 7a). Subsequently, the USP47-depleted cells and non-depleted cells were transfected with Flag-IK, and then treated with CHX. The USP47-depleted cells showed consistently reduced exogenous levels of IK (Fig. 7b). Next, to examine whether the degradation of IK in the USP47-depleted cells was mediated through the proteasomal pathway, the USP47-depleted cells were treated with the proteasome inhibitor, MG132. The IK degradation was impaired in the USP47-depleted cells treated with MG132 (Fig. 7c). In addition, the overexpression of USP47 increased the halflife of IK (Fig. 7d). To provide more direct evidence for the deubiquitination of IK by USP47, IK was immunoprecipitated from the lysates of USP47-depleted cells after MG132 treatment and then the level of ubiquitination was determined. The level of IK ubiquitination in the MG132- treated cells was greatly increased compared to that in the non-treated USP47-depleted cells (Fig. 7e). Moreover, forced expression of USP47 decreased the level of ubiquitinated-IK even after the MG132 treatment (Fig. 7f) and the expression of USP47 increased the level of IK in a dose-dependent manner (Fig. 7g).

\section{USP47 depletion decreases the level of ATM through IK degradation}

Because IK is associated with the proper splicing of ATM pre-mRNA, the ATM levels were examined in the USP47-depleted cells. Expectedly, the USP47-depleted cells which have low levels of IK showed lower levels of ATM compared to that in the non-depleted cells (Fig. 8a). Moreover, the ratio between intron retention/spliced isoforms of $A T M$ transcript at the junction of exons 1-2 was increased (Fig. 8b). To examine whether the aberrant splicing of ATM transcript at exons 1-2 could be rescued by restoration of IK, the USP47-depleted cells were transfected with IK-GFP, and successful ATM pre-mRNA splicing at exons 1-2 was observed (Fig. 8c). Moreover, the overexpression of IK partially rescued the low level of 


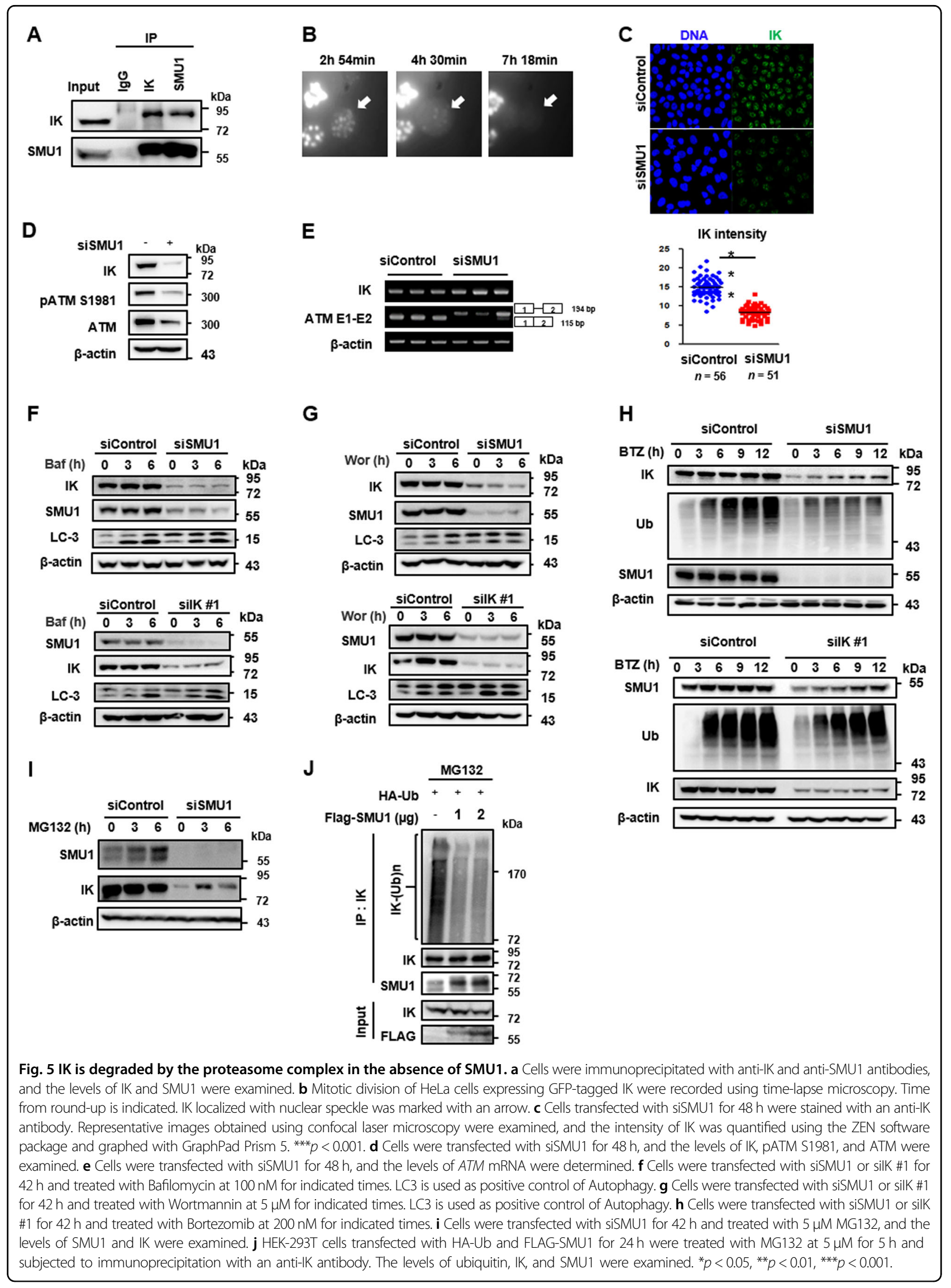




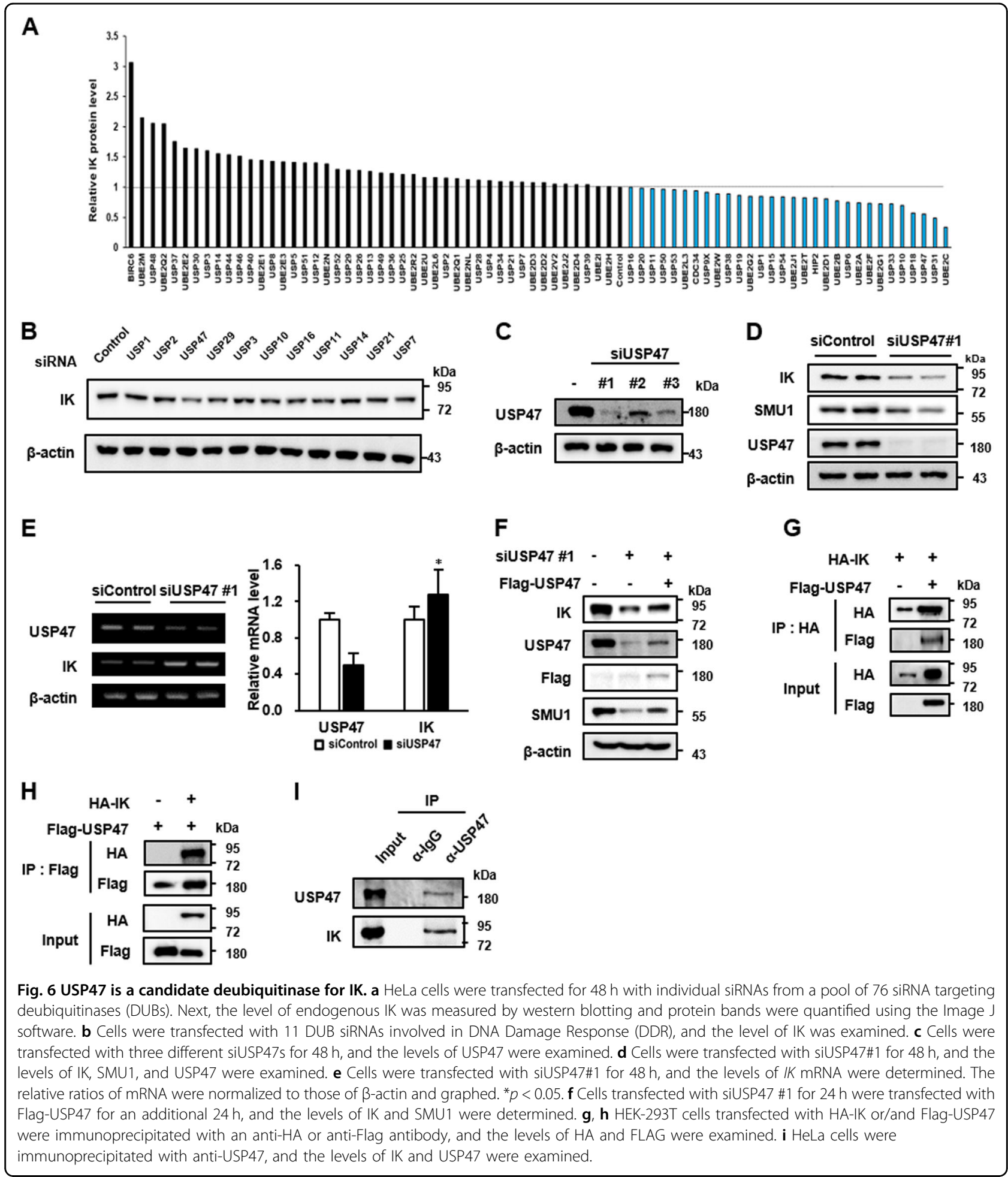

ATM protein upon USP47 depletion (Fig. 8d). Besides, to examine whether the USP47-depleted cells have an impairment of the ATM-mediated DDR, the USP47depleted cells were treated with HU for $24 \mathrm{~h}$ (Supplementary Fig. S4a). Similar to the result observed in the IK- depleted cells (Supplementary Fig. S1b), the USP47depleted cells showed a slight increase in ATM phosphorylation upon treatment of HU. It seems to be due to the decrease in the ATM protein levels upon IK depletion. Because the failure of ATM-mediated DDR increases 


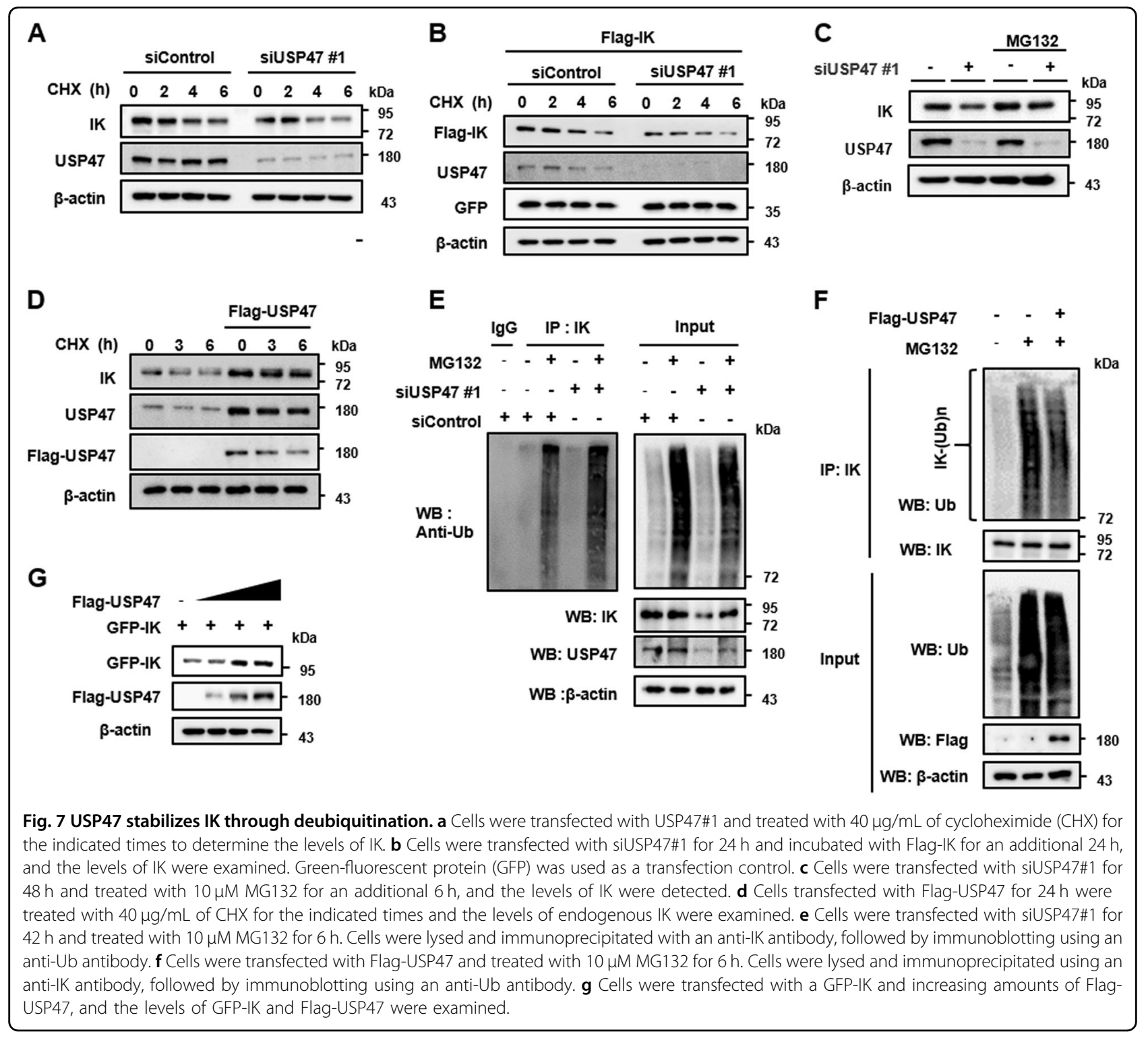

apoptosis, the USP47-depleted cells treated with HU were collected undergoing apoptosis in a living context. The HU-treated USP47-depleted cells showed an increase in cell death marker including cleaved PARP and cleaved caspase9 (Supplementary Fig. S4b) followed by a huge increase in apoptosis compared to that in the control cells (Supplementary Fig. S4c). Taken together, these results imply that USP47-mediated IK stabilization contributes to the ATM-mediated DDR through proper ATM premRNA splicing (Fig. 8e).

\section{Discussion}

The spliceosomal B-specific protein IK and SMU1, which are known to be involved in $B$ to $B^{\text {act }}$ transition, interact not only with each other but also with the U2 snRNP protein, SF3B3, and the RNA helicase, Brr2, to link the two proteins. Furthermore, knockdown of IK or SMU1 in human cells causes significant alternative splicing of many genes involved in cell death and survival ${ }^{33}$. During this work, Keiper et al. ${ }^{8}$ reported that IK and SMU1 play a critical role in the excision of short introns. In other words, introns retained after the knockdown of IK or SMU1 were predominantly shorter than $200 \mathrm{nt}$, whereas the majority of introns found in human cells are much longer than $200 \mathrm{nt}$. In the case of introns having a long distance between the 5 -SS and BS, the U2 domain is flexible enough to move toward Brr2. However, in the case of short introns ( $56 \mathrm{nt}$ or less), it is hard to move because of a structural constraint. To overcome this problem, IK and SMU1 extend the U2/Brr2 bridge. In this study, we showed that depletion of IK impaired the proper splicing of ATM pre-mRNA. Although ATM has many 


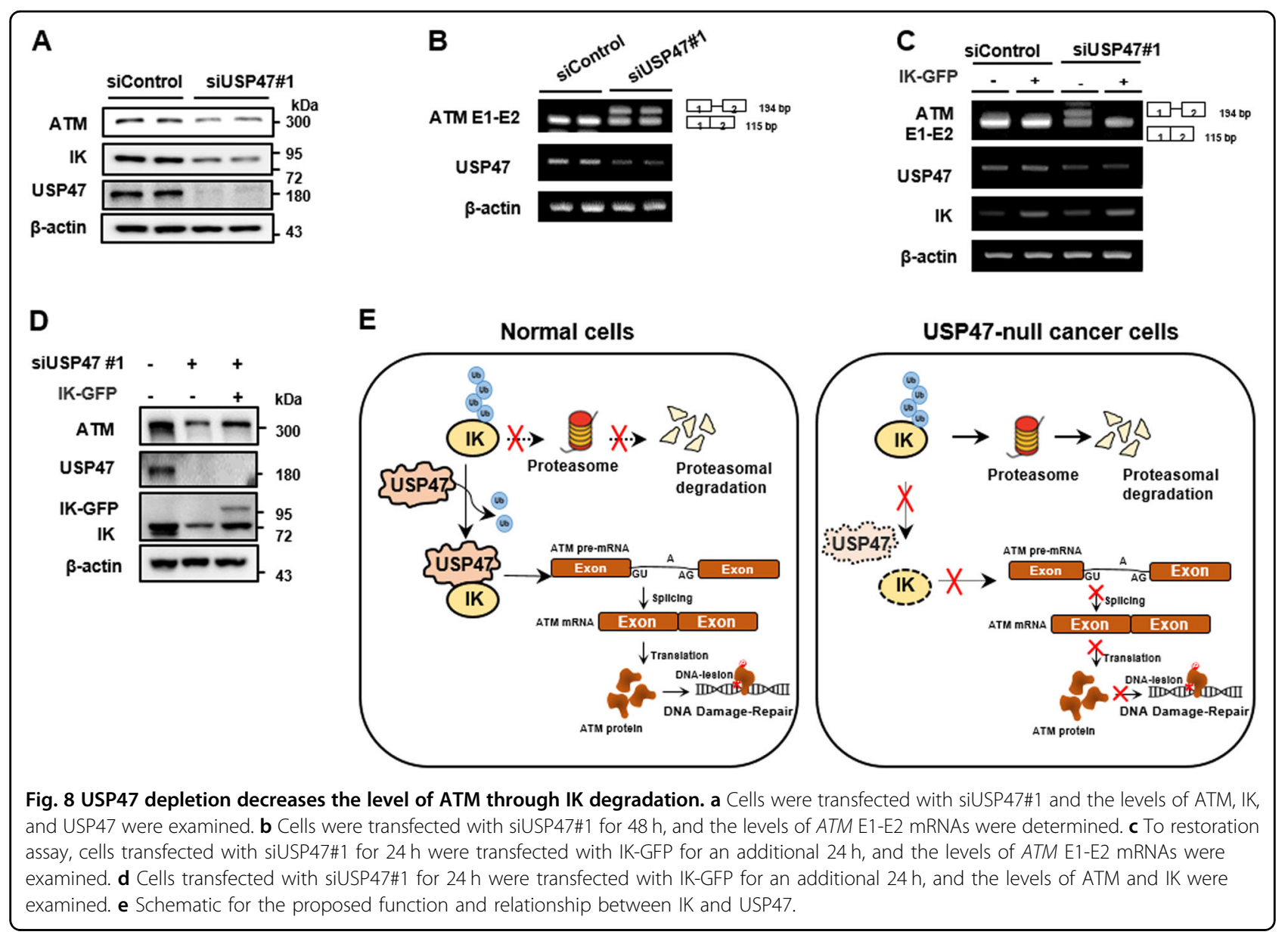

introns, only intron 1 was not spliced in absence of IK because it has a short length. This result clearly supports the fact that IK plays a critical role in excision of short introns.

In addition to the splicing function, several studies have shown that IK and SMU1 are also involved in genomic stability $^{34-37}$. One previous study showed that the loss of SMU1 function de-represses the DNA replication and over-activates the ATR-dependent replication checkpoint $^{36}$. Although IK depletion leads to decrease in SMU1 level, loss of IK did not phosphorylate CHK1 upon DNA damage. Next, we have examined the level of CHK1 phosphorylation in SMU1-depleted cells. The SMU1 depletion resulted in the phosphorylation of CHK1 upon NCS treatment (Supplementary Fig S5). Thus, it remains to uncover how reciprocal regulation of IK and SMU1 affect DDR independently.

The ATM protein signaling facilitates the repair of DNA damage in an early response to the DSBs ${ }^{38-42}$. The Mre11-Rad50-Nbs1 (MRN) complex autophosphorylates ATM after exposure to DNA damage ${ }^{43,44}$ which subsequently phosphorylates hundreds of substrates, including CHK1, CHK2, H2AX, p53, c-Abl, and
FANCD2 ${ }^{45}$, thus, leading to DNA repair and cell cycle arrest. In addition to ATM, the protein levels of the MRN complex were also decreased in IK-depleted cells (Supplementary Fig. S6a), but no change in the mRNA levels of Mre11, Rad50, and Nbs1 (Supplementary Fig. S6b). Because Mre11 and Rad50 genes do not have introns shorter than $200 \mathrm{bp}$, the decrease in protein levels was not because of the splicing failure of these mRNAs. In addition, the IK depletion-lowered ATM is not fully able to phosphorylate Nbs1 when activated by HU, which would weaken the Nbs1 and Mre11 interaction. The resulting Mre11 and Rad50 complex would be dysfunctional. Thus, it is likely that IK is involved in DDR through a proper splicing of ATM. On the other hand, ATM is also involved in DNA damage-induced spliceosome remodeling. The activated ATM reorganizes the distribution of spliceosomes on the pre-mRNA, thereby, causing dissociation of the spliceosomes from the pre-mRNA ${ }^{27,28}$. This spliceosome displacement results in the formation of R-loops at the DNA lesions, which alter $40 \%$ of the genome-wide UV-induced alternative splicing (AS) events. Therefore, the loss of the ATM protein in IKdepleted cells may concomitantly cause defects in the 
ATM-dependent spliceosome mobilization, thus, leading to wide-ranging changes in AS events.

IK/SMU1 is also involved in proper spindle attachment through interaction with MAD1 and causes cell cycle arrest at the mitotic phase. Other studies have demonstrated the connections between pre-mRNA splicing and the cell cycle ${ }^{46-49}$. In general, splicing is inhibited in extracts of mitotic cells, like the other steps in gene expression. Shin and Manley ${ }^{50}$ showed that the SR (rich in serine and arginine) protein, SRp38, represses the splicing during the mitotic phase through mitotic phase-specific dephosphorylation of SRp38. We also observed that IK localized at nuclear speckles and dissociated during mitotic phase (Supplementary Figs. S2 and 5b). Thus, splicing would be inhibited during mitotic phase through some other mechanism and IK released from nuclear speckle might play a role in the proper segregation of chromosomes. Hence, there should be a mechanism to keep IK from dissociation of nuclear speckle with independent functions and we show that USP47 may perform this role at the mitotic phase.

As DUBs modulate the half-life of many cellular proteins related to the signaling pathway, tumor suppression, tumorigenesis, immune response, and DDR, defects in DUBs induce various pathophysiological processes in the cell $^{51,52}$. Among the DUBs, many are associated with DDR. USP14 regulates the IR-induced NHEJ DNA repair $^{53}$. USP49 deubiqutinates p53 to prevent its degradation, and thus, USP49-depleted colon tumors have low levels of p53, which makes them more susceptible to DNA damage-inducers ${ }^{54}$. USP1 removes the monoubiquitination of PCNA, and thus UV-induced USP1 cleavage enables monoubiquitinated PCNA to accumulate and to activate translesion synthesis (TLS) ${ }^{55}$; as a result, USP1 knock-out mice are genetically unstable and hypersensitive to DNA damage. DNA damage-induced phosphorylation of USP10 moves it into the nucleus, allowing it to stabilize $\mathrm{p} 53^{56}$ and USP28 stabilizes Chk2 and 53BP1 in response to DNA damage ${ }^{57}$. USP47 is identified as a major deubiquitinase responsible for the stabilization of DNA polymerase $\beta$, which plays a critical role in DNA base excision repair ${ }^{58}$. In this study, we found that USP47 deubiquitinates the spliceosomal factor IK and associates with DDR through the complete splicing of ATM by IK stabilization.

Moreover, recent studies have revealed that pre-mRNA splicing depends on ubiquitination and deubiquitination cycles of the spliceosomal component. For example, the tri-snRNP proteins, PRP3 and PRP31, are reported to regulate the spliceosome through ubiquitination and deubiquitination ${ }^{17,59-61}$. First, the ubiquitin ligase, PRP19, containing the U-box spliceosomal protein, ubiquitinates PRP3 and PRP31, and then the ubiquitinated PRP3 and
PRP31 complex binds PRP8 and stabilizes the tri-snRNP complex. The ubiquitinated PRP3 and PRP31 are deubiquitinated by the ubiquitin-specific proteases, USP4 and USP15, respectively. It facilitates the ejection of U4 proteins from the spliceosome during maturation of its active site and progresses the splicing cycle. Ubiquitination plays a role as a mediator of protein-protein interaction in the above example and also functions in protein destabilization as for IK. For the proper spatio-temporal recruitment of IK into the spliceosomes, the stability of IK should be continuously regulated by the USP47 deubiquitinase. It needs to be investigated as to which E3 ligase ubiquitinates IK under a specific condition. In addition, the possibility that the ubiquitinated IK affects the modification of other spliceosomal complexes like PRP3 and PRP31 cannot be ruled out.

In summary, we found that IK depletion impairs the proper splicing of $A T M$ pre-mRNA at exons 1-2, which results in ATM deficiency followed by chromosome fragmentation and that USP47 directly binds to IK and deubiquitinates it leading to its stabilization. Thus, we provide the evidence that USP47 is indirectly involved in DDR through the complete splicing of ATM pre-mRNA by stabilization of the spliceosomal protein IK.

\section{Acknowledgements \\ Financial support was given by the National Research Foundation of Korea grant funded by the Korean government, MISP (Ministry of Science Information and Communication Technology, and Future Planning; SRC (Science Research Center) program (Cellular Heterogeneity Research Center: 2016R1A5A1011974). \\ Author details \\ 'Department of Biological Sciences, Sookmyung Women's University, Seoul 04310, Korea. ${ }^{2}$ Drug Evaluation Group, R\&D Center CJ HealthCare, Icheon 04551, Korea. 'Research Institute of Women's Health, Sookmyung Women's University, Seoul 04310, Korea. ${ }^{4}$ New Drug Development Center, Osong Medical Innovation Foundation, Osong 28160, Korea}

Conflict of interest

The authors declare that they have no conflict of interest.

\section{Publisher's note}

Springer Nature remains neutral with regard to jurisdictional claims in published maps and institutional affiliations.

The online version of this article (https://doi.org/10.1038/s41420-020-0268-1) contains supplementary material, which is available to authorized users.

Received: 16 February 2020 Revised: 24 March 2020 Accepted: 21 April 2020

Published online: 04 May 2020

\footnotetext{
References

1. Wahl, M. C., Will, C. L. \& Luhrmann, R. The spliceosome: design principles of a dynamic RNP machine. Cell 136, 701-718 (2009).

2. Dominguez, D. \& Burge, C. B. Interactome analysis brings splicing into focus. Genome Biol. 16, 135 (2015).
} 
3. Hegele, A. et al. Dynamic protein-protein interaction wiring of the human spliceosome. Mol. Cell 45, 567-580 (2012).

4. Zhou, Z., Licklider, L. J., Gygi, S. P. \& Reed, R. Comprehensive proteomic analysis of the human spliceosome. Nature 419, 182-185 (2002).

5. Lee, Y. \& Rio, D. C. Mechanisms and regulation of alternative pre-mRNA splicing. Annu. Rev. Biochem. 84, 291-323 (2015).

6. Bessonov, S., Anokhina, M., Will, C. L., Urlaub, H. \& Luhrmann, R. Isolation of an active step | spliceosome and composition of its RNP core. Nature 452 , 846-850 (2008).

7. Ulrich, A. K., Schulz, J. F., Kamprad, A., Schutze, T. \& Wahl, M. C. Structural basis for the functional coupling of the alternative splicing factors Smu1 and RED. Structure 24, 762-773 (2016).

8. Keiper, S. et al. Smu1 and RED are required for activation of spliceosomal B complexes assembled on short introns. Nat. Commun. 10, 3639 (2019).

9. Neumann, B. et al. Phenotypic profiling of the human genome by timelapse microscopy reveals cell division genes. Nature 464, 721-727 (2010).

10. Yeh, P. C., Yeh, C. C., Chen, Y. C. \& Juang, Y. L. RED, a spindle pole-associated protein, is required for kinetochore localization of MAD1, mitotic progression, and activation of the spindle assembly checkpoint. J. Biol. Chem. 287, 11704-11716 (2012).

11. Lee, S. et al. IK-guided PP2A suppresses Aurora B activity in the interphase of tumor cells. Cell. Mol. Life Sci. 73, 3375-3386 (2016).

12. Hu, L., Yang, F., Liu, X., Xu, D. \& Dai, W. Nuclear protein IK undergoes dynamic subcellular translocation and forms unique nuclear bodies during the cell cycle. Biomark. Res. 1, 11 (2013).

13. Lee, S., Han, S., Jeong, A. L., Park, J. S. \& Yang, Y. Depletion of IK causes mitotic arrest through aberrant regulation of mitotic kinases and phosphatases. FEBS Lett. 588, 2844-2850 (2014).

14. Will, C. L. \& Luhrmann, R. Spliceosome structure and function. Cold Spring Harb. Perspect. Biol. 3, 3707 (2011).

15. Shi, Y. Mechanistic insights into precursor messenger RNA splicing by the spliceosome. Nat. Rev. Mol. Cell Biol. 18, 655-670 (2017).

16. Bellare, P. et al. A role for ubiquitin in the spliceosome assembly pathway. Nat. Struct. Mol. Biol. 15, 444-451 (2008).

17. Das, T. et al. USP15 regulates dynamic protein-protein interactions of the spliceosome through deubiquitination of PRP31. Nucleic Acids Res. 45, 4866-4880 (2017).

18. Callis, J. The ubiquitination machinery of the ubiquitin system. Arabidopsis Book 12, e0174 (2014)

19. Glickman, M. H. \& Ciechanover, A. The ubiquitin-proteasome proteolytic pathway: destruction for the sake of construction. Physiol. Rev. 82, 373-428 (2002).

20. Lecker, S. H., Goldberg, A. L. \& Mitch, W. E. Protein degradation by the ubiquitin-proteasome pathway in normal and disease states. J. Am. Soc. Nephrol. 17, 1807-1819 (2006).

21. Guleria, A. \& Chandna, S. ATM kinase: much more than a DNA damage responsive protein. DNA Repair 39, 1-20 (2016).

22. Shiloh, Y. ATM and related protein kinases: safeguarding genome integrity. Nat. Rev. Cancer 3, 155-168 (2003).

23. Sancar, A., Lindsey-Boltz, L. A., Unsal-Kacmaz, K. \& Linn, S. Molecular mechanisms of mammalian DNA repair and the DNA damage checkpoints. Annu. Rev. Biochem. 73, 39-85 (2004).

24. Abraham, R. T. Cell cycle checkpoint signaling through the ATM and ATR kinases. Genes Dev. 15, 2177-2196 (2001).

25. Kastan, M. B. \& Lim, D. S. The many substrates and functions of ATM. Nat. Rev. Mol. Cell Biol. 1, 179-186 (2000).

26. Matsuoka, S. et al. ATM and ATR substrate analysis reveals extensive protein networks responsive to DNA damage. Science 316, 1160-1166 (2007).

27. Tresini, $M$. et al. The core spliceosome as target and effector of non-canonical ATM signalling. Nature 523, 53-58 (2015).

28. Tresini, M., Marteijn, J. A. \& Vermeulen, W. Bidirectional coupling of splicing and ATM signaling in response to transcription-blocking DNA damage. RNA Biol. 13, 272-278 (2016).

29. Katzenberger, R. J., Marengo, M. S. \& Wassarman, D. A. ATM and ATR pathways signal alternative splicing of Drosophila TAF1 pre-mRNA in response to DNA damage. Mol. Cell Biol. 26, 9256-9267 (2006).
30. Garner, E., Kim, Y., Lach, F. P., Kottemann, M. C. \& Smogorzewska, A. Human GEN1 and the SLX4-associated nucleases MUS81 and SLX1 are essential for the resolution of replication-induced Holliday junctions. Cell Rep. 5, 207-215 (2013).

31. Falck, J., Mailand, N., Syljuasen, R. G., Bartek, J. \& Lukas, J. The ATM-Chk2-Cdc25A checkpoint pathway guards against radioresistant DNA synthesis. Nature 410, 842-847 (2001).

32. Hong, S., Paulson, Q. X. \& Johnson, D. G. E2F1 and E2F3 activate ATM through distinct mechanisms to promote E1A-induced apoptosis. Cell Cycle 7, 391-400 (2008).

33. Papasaikas, P., Tejedor, J. R., Vigevani, L. \& Valcarcel, J. Functional splicing network reveals extensive regulatory potential of the core spliceosomal machinery. Mol. Cell 57, 7-22 (2015).

34. Sugaya, K., Hongo, E., Ishihara, Y. \& Tsuji, H. The conserved role of Smu1 in splicing is characterized in its mammalian temperature-sensitive mutant. J. Cell Sci. 119, 4944-4951 (2006).

35. Paulsen, R. D. et al. A genome-wide siRNA screen reveals diverse cellular processes and pathways that mediate genome stability. Mol. Cell 35, 228-239 (2009).

36. Ren, L. et al. Loss of Smu1 function de-represses DNA replication and overactivates ATR-dependent replication checkpoint. Biochem. Biophys. Res. Commun. 436, 192-198 (2013).

37. Sugaya, K., Hongo, E. \& Tsuji, H. A temperature-sensitive mutation in the WD repeat-containing protein Smu1 is related to maintenance of chromosome integrity. Exp. Cell Res. 306, 242-251 (2005).

38. Marechal, A. \& Zou, L. DNA damage sensing by the ATM and ATR kinases. Cold Spring Harb. Perspect. Biol. 5, 12716 (2013).

39. Khanna, K. K., Lavin, M. F., Jackson, S. P. \& Mulhern, T. D. ATM, a central controller of cellular responses to DNA damage. Cell Death Differ. 8, 1052-1065 (2001).

40. Rotman, G. \& Shiloh, Y. ATM: a mediator of multiple responses to genotoxic stress. Oncogene 18, 6135-6144 (1999).

41. Lee, J. H. \& Paull, T. T. Activation and regulation of ATM kinase activity in response to DNA double-strand breaks. Oncogene 26, 7741-7748 (2007).

42. Morrison, C. et al. The controlling role of ATM in homologous recombinational repair of DNA damage. EMBO J. 19, 463-471 (2000).

43. Lee, J. H. \& Paull, T. T. ATM activation by DNA double-strand breaks through the Mre11-Rad50-Nbs1 complex. Science 308, 551-554 (2005).

44. Uziel, T. et al. Requirement of the MRN complex for ATM activation by DNA damage. EMBO J. 22, 5612-5621 (2003).

45. Ahmed, K. M. \& Li, J. J. ATM-NF-kappaB connection as a target for tumor radiosensitization. Curr. Cancer Drug Targets 7, 335-342 (2007).

46. Blencowe, B. J. Splicing regulation: the cell cycle connection. Curr. Biol. 13, R149-R151 (2003).

47. Lundgren, $\mathrm{K}$. et al. A connection between pre-mRNA splicing and the cell cycle in fission yeast: cdc28+ is allelic with prp8+ and encodes an RNAdependent ATPase/helicase. Mol. Biol. Cell 7, 1083-1094 (1996).

48. Shea, J. E., Toyn, J. H. \& Johnston, L. H. The budding yeast U5 snRNP Prp8 is a highly conserved protein which links RNA splicing with cell cycle progression. Nucleic Acids Res. 22, 5555-5564 (1994).

49. Karamysheva, Z., Diaz-Martinez, L. A., Warrington, R. \& Yu, H. Graded requirement for the spliceosome in cell cycle progression. Cell Cycle 14, 1873-1883 (2015).

50. Shin, C. \& Manley, J. L. The SR protein SRp38 represses splicing in M phase cells. Cell 111, 407-417 (2002).

51. Komander, D. Mechanism, specificity and structure of the deubiquitinases. Subcell. Biochem 54, 69-87 (2010).

52. Mevissen, T. E. T. \& Komander, D. Mechanisms of deubiquitinase specificity and regulation. Annu. Rev. Biochem. 86, 159-192 (2017).

53. Sharma, A. et al. USP14 regulates DNA damage repair by targeting RNF168dependent ubiquitination. Autophagy 14, 1976-1990 (2018).

54. Tu, R. et al. USP49 participates in the DNA damage response by forming a positive feedback loop with p53. Cell Death Dis. 9, 553 (2018).

55. Huang, T. T. et al. Regulation of monoubiquitinated PCNA by DUB autocleavage. Nat. Cell Biol. 8, 339-347 (2006).

56. Yuan, J., Luo, K., Zhang, L., Cheville, J. C. \& Lou, Z. USP10 regulates p53 localization and stability by deubiquitinating p53. Cell 140, 384-396 (2010). 
57. Zhang, D., Zaugg, K., Mak, T. W. \& Elledge, S. J. A role for the deubiquitinating enzyme USP28 in control of the DNA-damage response. Cell 126, 529-542 (2006).

58. Parsons, J. L. et al. USP47 is a deubiquitylating enzyme that regulates base excision repair by controlling steady-state levels of DNA polymerase beta. Mol. Cell 41, 609-615 (2011).

59. Song, E. J. et al. The Prp19 complex and the Usp4Sart3 deubiquitinating enzyme control reversible ubiquitination at the spliceosome. Genes Dev. 24 1434-1447 (2010).
60. Hogg, R., McGrail, J. C. \& O'Keefe, R. T. The function of the NineTeen Complex (NTC) in regulating spliceosome conformations and fidelity during pre-mRNA splicing. Biochem. Soc. Trans. 38, 1110-1115 (2010).

61. Makarova, O. V., Makarov, E. M., Liu, S., Vornlocher, H. P. \& Luhrmann, R. Protein $61 \mathrm{~K}$, encoded by a gene (PRPF31) linked to autosomal dominant retinitis pigmentosa, is required for U4/U6*U5 tri-snRNP formation and pre-mRNA splicing. EMBO J. 21, 1148-1157 (2002). 